\title{
Numerical investigations of anisotropic structures of red blood cell aggregates on ultrasonic backscattering
}

Lenin Chinchilla, ${ }^{1}$ Curtis Armstrong, ${ }^{2}$ Rym Mehri, ${ }^{2}$ Alessandro S. Savoia, ${ }^{3}$ Marianne

Fenech, ${ }^{4}$ and Emilie Franceschini ${ }^{1, a)}$

${ }^{1}$ Aix-Marseille Univ., CNRS, Centrale Marseille, LMA UMR 7031, Marseille,

France

${ }^{2}$ University of Ottawa, Ottawa, Ontario, Canada

${ }^{3}$ Department of Engineering, Roma Tre University, 00146 Rome, Italy

${ }^{4}$ Department of Mechanical Engineering, University of Ottawa, Ottawa, Ontario, Canada

(Dated: 6 February 2021) 
Although quantitative ultrasound techniques based on the parameterization of the backscatter coefficient (BSC) have been successfully applied to blood characterization, theoretical scattering models assume blood as an isotropic scattering medium. However, the red blood cell (RBC) aggregates form anisotropic structures such as rouleaux. The present study proposes an anisotropic formulation of the Effective Medium Theory combined with the Local Monodisperse Approximation (EMTLMA) that considers perfectly aligned prolate-shaped aggregates. Theoretical BSC predictions were first compared with computer simulations of BSCs in a forward problem framework. Computer simulations were conducted for perfectly aligned prolate-shaped aggregates and more complex configurations with partially aligned prolate-shaped aggregates for which the size and orientation of RBC aggregates were obtained from blood optical observations. The isotropic and anisotropic EMTLMA models were then compared in an inverse problem framework to estimate blindly the structural parameters of RBC aggregates from the simulated BSCs. When considering the isotropic EMTLMA, the use of averaged BSCs over different insonification directions significantly improves the estimation of aggregate structural parameters. Overall, the anisotropic EMTLMA was found to be superior to the isotropic EMTLMA in estimating the scatterer volume distribution. These results contribute to a better interpretation of scatterer size estimates for blood characterization.

a)franceschini@lma.cnrs-mrs.fr 


\section{INTRODUCTION}

Quantitative UltraSound (QUS) techniques use the magnitude and frequency dependence of the backscatter spectrum from blood in order to obtain quantitative parameters reflecting the level of red blood cell (RBC) aggregation. A spectral analysis technique consists in estimating the spectral slope and intercept, ${ }^{1}$ and was applied to estimate the size of the blood microstructure and the acoustic concentration (i.e., the product of the scatterer concentration times the square of the relative impedance difference between scatterers and the surrounding plasma medium). ${ }^{2}$ Another QUS technique used to extract quantitative parameters from aggregated RBC structures relies on theoretical backscattering models in order to fit the measured backscatter coefficient (BSC) by a theoretical BSC. The challenge is to develop suitable theoretical backscattering models that consider the high volume fraction occupied by RBCs in blood (named hematocrit) and the clustering of RBCs characterized by specific size and shape. ${ }^{4}$ Two theoretical backscattering models have been developed: the Structure Factor Size Estimator (SFSE) and the Effective Medium Theory combined with the Structure Factor Model (EMTSFM). The SFSE theory approximates the structure factor with its second-order Taylor expansion and estimates two QUS parameters: the mean aggregate isotropic diameter and the packing factor. ${ }^{5,6}$ However, these two QUS parameters were found to be correlated, reducing the BSC parameterization to one QUS parameter and making the SFSE theory difficult to interpret physically. ${ }^{5,7}$ Despite this limitation, the SFSE theory was applied successfully to measure the RBC aggregation in relation to systemic inflammation in in vivo preclinical studies for varying circulatory disorders such 
as deep vein thrombosis, cardiopulmonary bypass and diabetes. ${ }^{8}$ The EMTSFM consists in treating the RBC aggregates as individual homogeneous scatterers and in calculating the backscattering from the collection of effective scatterers using the structure factor model. ${ }^{9}$ In the case of polydisperse aggregate size, the effective medium theory can be combined with the polydisperse structure factor model for a gamma size distribution, ${ }^{10}$ or combined with the Local Monodisperse Approximation (EMTLMA) for any size distribution. ${ }^{11}$ The local monodisperse approximation is an approximation of the polydisperse structure factor model, which is valid for moderate polydispersity. ${ }^{12}$ The polydisperse EMTSFM (or equivalently the polydisperse EMTLMA) provides three QUS parameters: the mean and standard deviation of the aggregate size distribution and the aggregate compactness. Previous three-dimensional (3D) computer simulations ${ }^{13}$ and in vitro experiments on aggregating blood $^{10,11}$ demonstrated that the EMTSFM and EMTLMA were more suitable than the SFSE for characterizing RBC aggregation. The EMTSFM and EMTLMA models provide a straightforward physical interpretation of the QUS estimates, since the aggregate size distributions estimated by these models are consistent with the direct optical observations in controlled blood flow. ${ }^{10,11}$

All the aforementioned models (SFSE, EMTSFM and EMTLMA) assume that aggregates are spherical, so that blood is considered as an isotropic scattering medium. However, it is well known that RBC aggregates form anisotropic structures, such as rouleaux in healthy blood or ellipsoidal clumps in pathological blood. ${ }^{14,15}$ Moreover, some in vitro ultrasonic studies on controlled blood flow reported angular-dependent backscatter intensity that sug- 
gest an orientation of the anisotropic aggregate structures with the flow streamlines. ${ }^{16,17,32} \mathrm{In}$ the presence of anisotropic aggregate structures, backscattering models considering spherical aggregates may create a bias against the QUS estimates.

The aims of the present study are:

- to propose an anisotropic formulation of the EMTLMA for modeling perfectly aligned prolate-shaped aggregates to better interpret anisotropic backscatter of aggregating blood, and

- to evaluate both isotropic and anisotropic EMTLMA to determine the structural features of anisotropic RBC aggregates from the measured BSCs.

There is no means to experimentally determine the RBC aggregate size and shape at a normal physiological hematocrit of $40 \%$, because blood is opaque to light. The use of optical methods to assess the RBC aggregate structures is limited to measurements in $2 \mathrm{D}$ confined flows $(<100 \mu \mathrm{m})$ and in cases of low hematocrits $(<20 \%){ }^{10,23,24}$ Whereas ultrasonic measurements of BSCs at $40 \mathrm{MHz}$, for instance, require the backscattered signal to be studied over an analysis window of at least 10 wavelengths $(\approx 385 \mu \mathrm{m})$, which is much larger than microchip or rheometer gap used to observe dynamics of RBC aggregates in optical microscopy. This is why only qualitative comparisons between ultrasonic and optical measurements have been carried out so far. ${ }^{5,10}$ The present study examines the anisotropic formulation of the EMTLMA and compares the use of this model to the isotropic EMTLMA from controlled computer simulations, where structural properties of RBC aggregates (size, 
shape, orientation) are known. Computer simulations were carried out for two different aggregating configurations: perfectly aligned prolate-shaped aggregates and more complex configurations with partially aligned prolate-shaped aggregates. The complex aggregation configurations correspond to the actual size and orientation distributions of aggregated RBCs obtained from optical measurements of sheared blood in a microfluidic chip. First, the BSC theoretical predictions given by the anisotropic EMTLMA are compared to simulated BSCs, which are obtained from 3D computer simulations in the framework of a forward problem study (i.e., the theoretical BSCs were determined from known structural properties of RBC aggregates). Second, the isotropic and anisotropic EMTLMA models are compared in the inverse problem framework to estimate blindly the structural parameters of RBC aggregates from the simulated BSCs. The ability of the two models (isotropic and anisotropic EMTLMA) as means of determining the aggregate size distribution is finally discussed.

\section{ULTRASOUND BACKSCATTERING THEORY}

\section{A. Differential backscattering cross-section modeling for a single aggregate}

The effective medium theory assumes that aggregates of RBCs can be treated as individual homogeneous scatterers, which have effective properties determined by the aggregate compactness $\phi_{i}$ and acoustic properties of plasma and RBCs. ${ }^{9,18}$ The aggregate compactness is defined as the volume fraction of RBCs within an aggregate. As a first approximation, it is assumed that all the deformable RBCs are tightly compact within the aggregates, so that the compactness $\phi_{i}$ is equal to 1 for all aggregates. Therefore, the acoustic properties 
of an aggregate (i.e., compressibility $\kappa$ and density $\rho$ ) are those of the RBCs. To consider the anisotropic structure of an aggregate, its shape is approximated by a prolate ellipsoid having a semi-minor axis $b$ and a semi-major axis $\nu b$ (with $\nu$ defined as the axial ratio). The differential backscattering cross section $\sigma$ of a prolate-shaped aggregate is given by: ${ }^{19}$

$$
\sigma(k)=\frac{k^{4}\left(\gamma_{\kappa}-\gamma_{\rho}\right)^{2}}{16 \pi^{2}} V^{2} F(k)
$$

where $k$ is the wavenumber, $V=\frac{4}{3} \pi \nu b^{3}$ the aggregate volume, $\gamma_{\kappa}$ the relative contrast in compressibility $\gamma_{\kappa}=\left(\kappa-\kappa_{0}\right) / \kappa_{0}$ and $\gamma_{\rho}$ the relative contrast in density $\gamma_{\rho}=\left(\rho-\rho_{0}\right) / \rho$, where $\kappa_{0}$ and $\rho_{0}$ are the compressibility and density of plasma, respectively. The ellipsoidal form factor $F(k)$ is defined as: ${ }^{20}$

$$
\begin{aligned}
& F(k)=\left(\frac{3(\sin (2 k u)-2 k u \cos (2 k u))}{(2 k u)^{3}}\right)^{2}, \\
& \text { with } \quad u=b \sqrt{1+\left(\nu^{2}-1\right) \cos ^{2} \theta},
\end{aligned}
$$

where $\theta$ is the relative orientation of ellipsoid, defined as the angle between the incident wave direction and the major axis of the prolate ellipsoid.

\section{B. Effective Medium Theory combined with the Local Monodisperse Approxima-}

\section{tion (EMTLMA)}

The effective medium theory is combined with the local monodisperse approximation to consider the interference effects caused by correlations between the spatial positions of effective prolate ellipsoids (i.e., the coherent scattering). ${ }^{11}$ As a first approximation, it is assumed that all the prolate ellipsoids have identical axial ratio $\nu_{f}$, that all the prolate ellipsoids are aligned in the same fixed direction $\theta_{f}$ and that their major axes are placed in 
the xy plane, as illustrated in Fig. 1. This configuration corresponds to aggregates aligned in the flow streamlines. The orientation angle $\alpha_{f}$ for a prolate ellipsoid is defined as the angle between its major axis and the $\mathbf{y}$ axis (rf. Fig. 1). One has $\alpha_{f}=\pi / 2-\theta_{f}-\beta$ so that either $\alpha_{f}$ or $\theta_{f}$ can be used to describe the prolate ellipsoid orientation.

The EMTLMA approximates the set of polydisperse prolate ellipsoids perfectly aligned by a set of non-interacting monodisperse subsystems. For each monodisperse subsystem, each scatterer with specific semi-minor axis $b$, axial ratio $\nu_{f}$ and orientation $\theta_{f}$ is assumed to be surrounded by scatterers with identical characteristics. Thus, the BSC is computed as the sum of the backscattering from monodisperse subsystem weighted by the probablity density function PDF of the semi-minor axis $b($ denoted $p(b))$ as:

$$
B S C_{\text {aniso }}(k)=m \int_{0}^{\infty} p(b) \sigma\left(k, b, \nu_{f}, \theta_{f}\right) S(k, u, \phi) \mathrm{d} b
$$

where $m$ is the number density of effective prolate ellipsoids, $\sigma$ is the differential backscattering cross-section given by Eq. (1), and $S$ is the monodisperse structure factor for an equivalent system, consisting only of particles of radius $u$ with a fixed total volume fraction $\phi$ occupied by the prolate ellipsoids. An analytical expression of the structure factor can be obtained by using the Percus-Yevick approximation as established by Wertheim ${ }^{21}$ and is given by Eqs. (A1)-(A4) in Ref. 20.

For the peculiar case of polydisperse aggregates with spherical shape, the BSC using the isotropic EMTLMA is expressed as:

$$
B S C_{\text {iso }}(k)=m \int_{0}^{\infty} p(r) \sigma(k, r) S(k, r, \phi) \mathrm{d} r
$$


where $\sigma(k, r)$ is the backscattering cross section of a spherical aggregate of radius $r$ given by Eq. (1) by using $\nu=1$ and $r=b$.

\section{MATERIAL AND METHODS}

\section{A. Experiments on human blood sheared in microfluidic shearing system}

Size and orientation distributions of RBC aggregates were obtained from experiments described in Refs. 23 and 24. These experiments are briefly summarized here. Two human blood samples (denoted A and B) were collected from two different healthy volunteers with the approval of the ethics committee of the University of Ottawa (H11-13-06). The blood was prepared following standard procedures as previously described. ${ }^{24}$ Human RBCs were then re-suspended in their respective plasma at an hematocrit of $10 \%$. The RBC suspensions were flowed and observed in vitro in a two-fluid flow poly-di-methyl-siloxane (PDMS) microfluidic shearing system (see Fig. 1 in Ref. 24). The channel dimension is $120 \mu \mathrm{m}$ in width and $60 \mu \mathrm{m}$ in height. Within this system, the RBC aggregates were entrained using a second solution of phosphate buffered saline in order to obtain a linear velocity profile within the blood layer and thus achieve a wide range of constant shear rates. The shear rate was determined using a micro Particle Image Velocimetry ( $\mu$ PIV) system (FlowMaster MITAS, LaVision, USA), while RBC aggregates were simultaneously visualized by bright field microscopy and recorded with a high speed camera (Basler acA2000-340kmNIR). Finally, the contours of approximately 400 aggregates were segmented manually on several 
images. Each RBC aggregate contour is then fitted by an ellipse to obtain its semi-minor axis, semi-major axis and orientation.

\section{B. Computer simulations based on the Structure Factor Model}

Computer simulations based on the Structure Factor Model (SFM) were conducted to obtain the simulated $B S C_{\text {sim }}$ from different aggregating configurations. First, polydisperse prolate ellipsoids perfectly aligned were simulated, as illustrated in Fig. 1. The prolate ellipsoids have identical axial ratio $\nu_{f}$ and their semi-minor axes $b$ are gamma distributed in line with the anisotropic EMTLMA. For a fixed mean semi-minor axis $\bar{b} / a=2.5$, we study several structural configurations with varying axial ratios $\nu_{f}$ (ranging from 1.5 to 2.5) and varying semi-minor axis standard deviations $\bar{b}$ (ranging from $0.18 a$ to $0.75 a$ ). Second, the complex aggregate configurations were based on optical measurements obtained from microfluidic experiments as described in subsection III A. The RBC aggregates were approximated by prolate ellipsoids with semi-minor axes, axial ratios and orientations following the distributions of size and orientation obtained from optical image segmentation of RBC aggregates.

For each aggregation configuration studied, non-overlapping prolate ellipsoids (i.e., nonoverlapping RBC aggregates) were randomly distributed in a simulated volume $V_{\text {sim }}$ using a Monte Carlo algorithm (see section II.B.A in Ref. ${ }^{27}$ ). The simulated volume is fixed to 250 $\times 250 \times 250 \mu \mathrm{m}^{3}$, and the hematocrit is fixed to $10 \%$ or $30 \%$. The simulated $B S C_{\text {sim }}$ was obtained using the SFM as follows:

$$
B S C_{\text {sim }}(k)=m\left\langle\frac{1}{N}\left|\sum_{j=1}^{N} \Phi(k) e^{-i 2 k \mathbf{n} \cdot \mathbf{r}_{j}}\right|^{2}\right\rangle
$$


where $m$ is the number density of prolate ellipsoids equal to $N / V_{\text {sim }}, \Phi$ is the backscattering amplitude of $j$ th prolate ellipsoid, $\mathbf{r}_{j}$ is the location of the $j$ th prolate ellipsoid and $\mathbf{n}$ is the unit vector in the direction of the incident field. The symbol \langle\rangle represents the ensemble average. The total number of prolate ellipsoids $N$ is calculated by forcing the prescribed volume fraction to $\phi=\left(\sum_{j=1}^{N} \frac{4 \pi}{3} \nu_{j} b_{j}^{3}\right) / V_{\text {sim }}$. The backscattering amplitude $\Phi$ is defined as $\Phi^{2}(k)=\sigma(k)$, where $\sigma$ is given in Eq. (1). One simulated $B S C_{\text {sim }}$ was computed by averaging 100 realizations for averaging purposes.

The simulated $B S C_{\text {sim }}$ were performed with thirteen different insonification angles $\beta$ ranging from $-30^{\circ}$ to $30^{\circ}$ with a step of $5^{\circ}$. In the case of perfectly aligned prolate ellipsoids, the insonification angle $\beta=0^{\circ}$ corresponds to the angle for which the major axis direction of the prolate ellipsoids and the incident field direction are perpendicular. (This specific direction of the major axis of the aligned prolate ellipsoids was arbitrarily chosen.) Examples are shown in Fig. 2(a), where simulated $B S C_{\text {sim }}$ were calculated for perfectly aligned prolate ellipsoids $\left(\phi=30 \%, \bar{b} / a=2.5, \sigma_{b} / a=0.75, \nu_{f}=2\right)$ for different insonification angles $\beta$. A change of BSC magnitude regarding different insonification angles $\beta$ can be observed.

\section{QUS parameter estimation}

The measured BSCs with different insonification angles bring information on the anisotropy of the scattering medium. For each insonification angle $\beta$, the $i B S C$ is computed in the wavenumber range of $k_{1}$ to $k_{2}$ as follows:

$$
i B S C(\beta)=\frac{1}{\left(k_{2}-k_{1}\right)} \int_{k_{1}}^{k_{2}} 10 \log _{10}(B S C(k, \beta)) d k
$$


The example of $i B S C$ given in Fig. 2(b) suggests that the $i B S C$ is highest when the acoustic beam is perpendicular (normal) to the aligned structures, and the magnitude of $i B S C$ symmetrically decreases about the angle of normal incidence. ${ }^{28}$ We evaluate the level of anisotropy by measuring the difference between the maximum and minimum values of $i B S C(\beta)$, denoted $\mathrm{D}_{i B S C}$ (rf. Fig. 2(b)). The orientation $\theta_{f}$ of the aligned structures can be obtained by fitting a quadratic function $h(\beta)=c_{2} \beta^{2}+c_{1} \beta+c_{0}$ to the measured $i B S C(\beta)$ (rf. Fig. 2(b)). The location of the axis of symmetry of the quadratic function at $\beta_{0}=-c_{1} /\left(2 c_{2}\right)$ gives the incident wave direction perpendicular to the axis of alignment of the ellipsoids. It can be deduced that $\alpha_{f}=-\beta_{0}$, or equivalently $\theta_{f}=\pi / 2-\alpha_{f}-\beta$.

The QUS structural parameters are estimated by fitting the simulated $B S C_{\text {sim }}$ with the theoretical EMTLMA, and by assuming that the hematocrit $\phi$, the RBC radius $a$ and the acoustical properties of plasma and RBCs are known a priori. The parameter estimation was carried out in the 10-42 MHz frequency bandwidth, which corresponds to the frequency range used in a previous in vitro experimental study. ${ }^{10}$ The main orientation of the aligned structures is first determined as described previously, and the unknown parameters (mean semi-minor axis $\bar{b}^{*}$ with standard deviation $\sigma_{b}^{*}$, and axial ratio $\nu_{f}^{*}$ ) are determined by minimizing the cost function $\mathcal{F}$, which synthesizes the thirteen simulated $B S C_{\text {sim }}$ with the thirteen insonification angles over the wavenumbers $k_{j}$ in the wavenumber range of $k_{1}$ to $k_{2}$ :

$$
\mathcal{F}=\frac{1}{M} \sum_{i=1}^{M} \frac{\sum_{j}\left\|B S C_{\text {sim }}\left(k_{j}, \theta_{i}\right)-B S C_{\text {aniso }}\left(k_{j}, \theta_{i}\right)\right\|^{2}}{\sum_{j}\left\|B S C_{\text {sim }}\left(k_{j}, \theta_{i}\right)\right\|^{2}}
$$

where $M$ is the number of insonification angles (here $M=13$ ) and $B S C_{\text {aniso }}$ corresponds to the anisotropic EMTLMA given by Eq. (3). 
Finally, the isotropic EMTLMA was also employed to assess its ability of evaluating the QUS parameters in anisotropic aggregating configurations. In that case, the isotropic EMTLMA is fit to one single simulated $B S C_{\text {sim }}$ : the $B S C_{\text {sim }}$ averaged over the thirteen insonification angles (denoted $\overline{B S C(\beta)}$ ), or the single $B S C_{\text {sim }}$ at the insonification angle $\beta=0^{\circ}$. The unknown parameters (mean radius $\bar{r}^{*}$ with standard deviation $\sigma_{r}^{*}$ ) are determined by minimizing the relative mean error between one single $B S C_{\text {sim }}$ and the theoretical $B S C_{\text {iso }}$ given by Eq. (4).

Whatever the model used (isotropic or anisotropic), the routine fmincon of MATLAB was employed to minimize the cost function $\mathcal{F}$, with the constraint $p\left(r_{\mathrm{eq}}<a_{\mathrm{min}}\right)=0$ (i.e., the equivalent aggregate radius should be larger than $\left.a_{\min }=1.82 \mu \mathrm{m}\right)$. The value $a_{\min }$ corresponds to the minimum radius of sphere having the equivalent volume of a $\mathrm{RBC}$ in the segmented images shown in Fig. 4(a). (This value is discussed in the Section V C.)

\section{RESULTS}

\section{A. Forward problem: comparison between simulated and theoretical BSCs}

The aim was to compare the simulated $B S C_{\text {sim }}$ and the theoretical $B S C_{\text {theo }}$ in a large frequency bandwidth of 10-70 MHz using the EMTLMA in the framework of the forward problem study (i.e., determining the BSCs from known distributions of aggregate shape, size and orientation using the SFM and EMTLMA). The relative error in $B S C_{\text {theo }}$ was calculated over the 10-70 MHz frequency range as follows: $\epsilon(k)=\left\|B S C_{\text {sim }}(k)-B S C_{\text {theo }}(k)\right\| / B S C_{\text {sim }}(k)$. 


\section{Perfectly aligned prolate ellipsoids}

Polydisperse aligned prolate ellipsoids were studied with identical mean semi-minor axis $\bar{b} / a=2.5$, and varying $\sigma_{b} / a=0.18,0.35$ or 0.75 , and $\nu_{f}=2.0$ or 2.5 (rf. Fig. 3). As $\nu_{f}$ increases, the volume of scatterers increase, and, as a consequence, the $B S C_{\text {sim }}$ amplitude increases at low frequencies (before the first peak occurrence) and the first peak of the $B S C_{\text {sim }}$ occurs at lower frequencies (rf. Fig. 3(a)). As $\sigma_{b} / a$ increases, the $B S C_{\text {sim }}$ peaks are less pronounced and the $B S C_{\text {sim }}$ amplitude increases at low frequencies (rf. Fig. 3(b)).

When the $B S C_{\text {sim }}$ are compared angle by angle, the first peak of the $B S C_{\text {sim }}$ occurs at higher frequencies when $\sigma_{b} / a$ decreases, as expected (rf. Fig. 3(b)). For instance, for the insonification angle $\beta=30^{\circ}$, the frequency at which the first peak of the $B S C_{\text {sim }}$ occurs is 34 MHz with $\sigma_{b} / a=0.75$, and is $40 \mathrm{MHz}$ with $\sigma_{b} / a=0.18$.

It is noticed that the dynamic range of BSC magnitude increases as the axial ratio $\nu_{f}$ increases (rf. Figs. 3(a) and 3(c)). The changes in BSC magnitude at different insonification angles are well pronounced in the high frequencies $(>42 \mathrm{MHz})$ in all the cases studied, but these changes are less pronounced in lower frequencies as the polydispersity decreases (rf. Fig. 3(b)). Therefore, the difference between the maximum and minimum values of $i B S C(\beta)$ $\left(\mathrm{D}_{i B S C}\right)$ is less than $0.46 \mathrm{~dB}$ for $\sigma_{b} / a \leq 0.45$, against $\mathrm{D}_{i B S C} \approx 1.49 \mathrm{~dB}$ for $\sigma_{b} / a=0.75$ (rf. Fig. $3(\mathrm{c}))$. One can observe that, in the majority of the cases studied, the $i B S C$ is highest at $\beta=0$, when the acoustic beam is perpendicular to the aligned structures (rf. Fig. 3(c)). In the case $\left(\sigma_{b} / a=0.18, \nu_{f}=2.0\right)$, the $i B S C$ is lowest at $\beta=0^{\circ}$ because the peaks of $B S C_{\text {sim }}$ 
occurs at higher frequencies for the smallest $\sigma_{b} / a$, so the $B S C_{\text {sim }}$ are similar to within 0.5 $\mathrm{dB}$ for frequencies less than $42 \mathrm{MHz}$.

Also plotted in Figs. 3(a) and 3(b) are the theoretical $B S C_{\text {aniso }}$ computed with the anisotropic EMTLMA given by Eq. (3) (solid lines). Satisfactory agreement was found between the simulation and the anisotropic EMTLMA theory with relative errors in $B S C_{\text {aniso }}$ less than $22 \%$, whatever the aggregating configuration studied for aligned prolate ellipsoids.

\section{Complex aggregating configuration}

A typical example of RBC aggregates flowing in two-fluid flow microfluidic shearing system, together with the corresponding contour segmentation, is shown in Fig. 4(a). Histograms of the distributions of the semi-minor axis, axial ratio, orientation and equivalent radius are represented in Figs. 4(b)-4(e) for both blood samples A and B. The equivalent radius $r_{\text {eq }}$ corresponds to the sphere radius of equivalent volume to that of prolate ellipsoid. The semi-minor axis $b$ and the equivalent radius of aggregate $r_{\text {eq }}$ are normalized by the characteristic radius of a single $\mathrm{RBC} a=2.75 \mu \mathrm{m}$. These histograms correspond to the highest levels of aggregation obtained from each blood sample and were used as input data to obtain the simulated $B S C_{\text {sim }}$.

Figures (5a) and (5b) compares the simulated $B S C_{\text {sim }}$ and the theoretical $B S C_{\text {aniso }}$ when considering the complex aggregating configuration for the blood sample A at hematocrits of $10 \%$ and $30 \%$. In line with the anisotropic EMTLMA proposed in section II B, the theoretical $B S C_{\text {aniso }}$ considers the average axial ratio $\overline{\nu_{f}}=2.7$ and the main aggregate orientation $\alpha_{f}=-$ $\beta_{0}=-7^{\circ}$ for $\phi=10 \%$ (and $\alpha_{f}=-8^{\circ}$ for $\phi=30 \%$ ). The main orientation $\alpha_{f}$ was estimated by 
fitting the $i B S C$ as a function of the insonification angle $\beta$ (rf. Fig. 5(c)), and agrees well with the maximum value of the histogram distribution of orientation (rf. Fig. 4). One can observe that the theoretical anisotropic EMTLMA produces an overestimation of the dynamic range of BSC magnitude when compared to the simulated $B S C_{\text {sim. }}$. This discrepancy will be discussed later in the Section V A.

\section{B. Inverse problem: estimation of QUS parameters}

In the following subsection, each given QUS parameter is the average over 10 QUS parameters estimated from 10 simulated $B S C_{\text {sim }}$ in the same aggregating configuration. The goodness-of-fit of the theoretical EMTLMA to the simulated $B S C_{\text {sim }}$ was assessed by the coefficient of determination.

Table II gives the mean values of QUS parameters estimated from the anisotropic and isotropic EMTLMA for the aggregating configurations consisting in aligned prolate ellipsoids. Also given in Table II are the $\mathrm{D}_{i B S C}$ and the goodness-of-fit statistic $\mathrm{R}^{2}$. The goodness-of-fit statistic $\mathrm{R}^{2}$ reveals that the anisotropic EMTLMA provides the best fit to the $B S C_{\text {sim }}$ curves $\left(\mathrm{R}^{2} \geq 0.97\right)$. For the largest polydispersity with $\sigma_{b} / \mathrm{a}=0.75$, the isotropic EMTLMA gives poorer fit curves with $\mathrm{R}^{2}$ comprised between 0.25 and 0.93 . The smallest value of $\mathrm{R}^{2}=0.25$ was obtained for the largest axial ratio $\nu_{f}=2.5$, corresponding to the largest value of $\mathrm{D}_{i B S C}$. When considering the isotropic EMTLMA using $\overline{B S C(\beta)}$, the mean $r_{\text {eq }}^{*} / a$ and $\sigma_{\text {eq }}^{*} / a$ were estimated with relative errors less than $9 \%$ and $30 \%$, respectively (against relative errors up to $16 \%$ and $70 \%$ when considering the isotropic EMTLMA using $\left.B S C\left(\beta=0^{\circ}\right)\right)$. When considering the anisotropic EMTLMA, the mean of QUS parameters 
matched well the actual structural parameters with relative errors less than $3 \%$ for $\bar{b} / a, 9 \%$ for $\sigma_{b} / a$ and $4 \%$ for $\nu_{f}$, by excluding the case of aligned prolate ellipsoids with $\sigma_{b} / a=0.35$ and $\nu_{f}=2$ (rf. Table II line 5). For this particular case, which corresponds to the smallest level of anisotropy (i.e., the smallest value of $\mathrm{D}_{i B S C}$ ), the relative errors of the estimates are higher: up to $20 \%$ for the estimated $\sigma_{b}^{*} /$ a. Figure 6 gives some examples of histograms of $r_{\text {eq }}^{3} p\left(r_{\text {eq }}\right)$ estimated by the anisotropic or isotropic EMTLMA. The histograms of $r_{\text {eq }}^{3} p\left(r_{\text {eq }}\right)$ are represented because the backscattering amplitude is proportional to the scatterer volumes (and not the scatterer sizes). Overall, the aggregate volume distributions estimated by the anisotropic EMTLMA match better the actual volume distribution when compared to the isotropic EMTLMA.

Table III gives the mean values of QUS parameters estimated from the anisotropic and isotropic EMTLMA for hematocrits of $10 \%$ and $30 \%$ when considering the complex aggregating configurations with blood samples A and $\mathrm{B}$. The isotropic EMTLMA using $\overline{B S C(\beta)}$ had the best fit to the data with goodness-of-fit statistics $R^{2} \geq 0.95$, against the anisotropic EMTLMA giving $\mathrm{R}^{2}$ comprised between 0.85 and 0.93 . When considering the isotropic EMTLMA using $\overline{B S C(\beta)}$, mean sizes and standard deviations were estimated with relative errors less than $18 \%$ and $8 \%$, respectively (against relative errors less than $8 \%$ and $58 \%$ when considering the isotropic EMTLMA using $\left.B S C\left(\beta=0^{\circ}\right)\right)$. When considering the anisotropic EMTLMA, the estimated equivalent radii were also computed as $r^{*}=b^{*} \sqrt[3]{\nu_{f}^{*}}$. In that case, the mean size and standard deviation of $r^{*} / a$ were estimated with relative errors less than $13 \%$ and $9 \%$, respectively. Overall, the anisotropic EMTLMA largely underestimates the mean axial ratio $\bar{\nu}$ with relative errors up to $93 \%$. Examples of histograms of $r_{\text {eq }}^{3} p\left(r_{\text {eq }}\right)$ 
are given in Fig. (7). The scatterer volume PDFs estimated by the anisotropic EMTLMA and by the isotropic EMTLMA using $\overline{B S C(\beta)}$ are similar. Note that, in the case of the blood sample $\mathrm{B}$, the value of $\mathrm{D}_{i B S C}$ is the smallest (less than $0.75 \mathrm{~dB}$ ), and the anisotropic EMTLMA estimates an axial ratio $\nu_{f}$ equal to 1 (i.e., isotropic medium). That is why the isotropic and anisotropic models gave identical scatterer volume PDF in that case (rf. Fig. $7(\mathrm{~b}))$

Note that the mean and standard deviation of the QUS parameters are given in supplemental Tables ${ }^{29}$ for all aggregating configurations studied.

\section{DISCUSSION AND CONCLUSION}

\section{A. On the assumption of perfectly aligned aggregates when using the anisotropic}

\section{EMTLMA}

The anisotropic EMTLMA was developed to model perfectly aligned prolate ellipsoids with identical axial ratio. Based on this modeling, the angle dependent BSCs from aggregated RBCs were parameterized by four QUS parameters: the orientation $\alpha_{f}$, the axial ratio $\nu_{f}$, and the mean $\bar{b}^{*}$ and standard deviation $\sigma_{b}^{*}$ of the semi-minor axis PDF. Both assumptions of identical axial ratio and perfect alignment of aggregates allows us to reduce the number of unknown QUS parameters, but they do not reflect the reality of rouleaux structures as observed in the histograms of Figs. 4(c) and 4(d). Therefore, the anisotropic EMTLMA modeling is not sufficient to model the backscattering from complex anisotropic aggregates, as shown previously Figs. 5(a) and 5(b). 
To better understand these discrepancies, it is necessary to study the effect of fixed axial ratio $\nu_{f}$ and/or fixed aggregate orientation $\alpha_{f}$ on the BSC. In that aim, additional computer simulations were performed to compute the $B S C_{\text {sim }}$ from blood sample $\mathrm{A}$ at hematocrit $\phi=10 \%$ by considering the joint PDF of $b, \nu$ and $\alpha$ with a fixed $\nu_{f}=2.7$ (denoted $\left.p\left(b, \nu_{f}, \alpha\right)\right)$ and/or with a fixed $\alpha_{f}=7^{\circ}\left(\right.$ denoted $\left.p\left(b, \nu, \alpha_{f}\right)\right)$. Figure 8 compares simulated $B S C_{\text {sim }}$ by using the aggregate features of blood sample $\mathrm{A}$ and by considering different joint PDFs: $p(b, \nu, \alpha), p\left(b, \nu_{f}, \alpha\right), p\left(b, \nu, \alpha_{f}\right)$ and $p\left(b, \nu_{f}, \alpha_{f}\right)$. Note that the joint PDF $p(b, \nu, \alpha)$ gives the true simulated $B S C_{\text {sim }}$ (as already plotted in Fig. 5) and serves as reference data. One can observe that the fixed $\alpha_{f}$ produces a large overestimation of dynamic range of the $B S C_{\text {sim }}$ magnitude, whereas the fixed $\nu_{f}$ does not produce a significant change in the $B S C_{\text {sim }}$ magnitude. Similar observations were obtained for the blood sample B (data not shown). Therefore, the assumption of perfectly aligned prolate-shaped aggregate mainly causes the discrepancies between simulated $B S C_{\text {sim }}$ and theoretical $B S C_{\text {aniso }}$ for the two blood samples considered in this study.

In addition, this extensive forward problem study allows us to better understand the estimates of QUS parameters. Indeed, the axial ratio $\nu_{f}$ is always largely underestimated, as shown in Table III. Let recall that the decrease in $\nu_{f}$ has the effect of decreasing the $\mathrm{D}_{i B S C}$, as shown in Fig. 3(a). Since the assumption of a fixed $\alpha_{f}$ has the effect of overestimating the $\mathrm{D}_{i B S C}$, it goes with the underestimation of $\nu_{f}$. 


\section{B. QUS parameters estimated by the isotropic and anisotropic EMTLMA}

When considering perfectly aligned prolate-shaped aggregates, the anisotropic EMTLMA was found to be superior to the isotropic EMTLMA for characterizing scatterer volume distribution (rf. Fig. 6). The large differences between actual and estimated QUS parameters were obtained when the isotropic EMTLMA using $B S C\left(\beta=0^{\circ}\right)$ was used to fit the measured data. However, it is interesting to observe that the mean scatterer radius matches quite well the expected radii (with relative errors less than 9\%) when using the isotropic EMTLMA with $\overline{B S C(\beta)}$.

When considering the complex aggregating configurations with blood sample A, the anisotropic EMTLMA estimates the scatterer volume distribution more accurately than the isotropic EMTLMA (rf. Table III and Fig. 7). In that case, the mean radius $\bar{r}^{*} / a$ underestimates the actual $\bar{r}_{\text {eq }} / a$ with relative errors up to $13 \%$ when using the anisotropic EMTLMA, against relative errors up to $18 \%$ when using the isotropic EMTLMA. This underestimation of scatterer radius may be caused by the partial alignement of rouleaux, since the rouleaux structures are neither randomly oriented nor perfectly aligned.

Both anisotropic EMTLMA and isotropic EMTLMA using $\overline{B S C(\beta)}$ yield quite similar scatterer volume distribution when considering complex aggregating configurations. Although the mean radius is slightly better estimated by the anisotropic EMTLMA, further aggregating configurations (with more varied sizes and orientations) need to be performed to evaluate the added value of anisotropic modeling. At present, the isotropic modeling (EMTLMA or EMTSFM) is fairly simple to implement and has proven its effectiveness in 
estimating the scatterer volume distribution in previous experimental works on aggregating blood. $^{10,11}$

\section{Towards more realistic aggregating configurations in simulation}

In previous numerical studies, the ultrasonic backscattering of anisotropic RBC aggregates was revealed by using simulated spatial arrangements of RBCs obtained from particle dynamics $^{25}$ or statistical mechanics. ${ }^{26}$ In those two-dimensional (2D) simulations, the size and shape of RBC aggregates depend on the contribution of adhesive, repulsive and shear forces set in the mechanical models. Although the resulting shapes visually resemble aggregate of porcine RBCs, the elongation and orientation of anisotropic aggregates observed in human blood cannot be reproduced with such simple models. ${ }^{30}$ In the present study, the structural characteristics (i.e. size, shape, orientation) of RBC aggregates were obtained from optical measurements of blood sheared in a microfluidic chip. Then prolate-shaped aggregates having these structural characteristics were randomly distributed within the simulated volume to compute the angular-dependent $B S C_{\text {sim }}$. To our knowledge, this is the first time that actual size and orientation distributions of RBC aggregates obtained from experiments are used for numerical simulations to study anisotropic backscatter in aggregating blood. However, the actual aggregate structures obtained in our microfluidic experiment may differ from realistic conditions encountered in large blood vessels $(\approx 1 \mathrm{~mm})$ at physiological hematocrit, because of the experimental constraints for obtaining aggregate structures by optical techniques as detailed below. First, the optical measurements were limited to a low hematocrit of $\phi=10 \%$, because the RBCs are opaque to light. This opacity prevents 
optical measurements at physiological hematocrit of $30-50 \%$. So the simulations performed at $\phi=30 \%$ were based on the distributions of aggregate size and orientation measured at $\phi=10 \%$, whereas these distributions are expected to be different at $\phi=10 \%$ and $30 \%$. Second, the two-fluid microfluidic shearing system has a small channel dimension of $120 \mu \mathrm{m} \times$ $60 \mu \mathrm{m}$, so aggregate sizes in this shearing system may be smaller than those encountered in large blood vessels $(\approx 1 \mathrm{~mm})$. Third, the optical measurements give only access to twodimensional (2D) images of aggregates, so the aggregate shape was approximated by prolate ellipsoid deduced from the fitted ellipse (see Fig. 4) and its major axis is assumed to be placed in the xy plane. This extrapolation of 2D information in 3D may also result in biases on the polydispersity of the shape and orientation of the aggregates. For example, a single $\mathrm{RBC}$ in 3D space is like an oblate ellipsoid of radii $4.2 \mu \mathrm{m}, 4.2 \mu \mathrm{m}$ and $1.2 \mu \mathrm{m}$ (having the equivalent volume of a sphere of radius $a=2.75 \mu \mathrm{m}$ ), whereas the single RBC seen on the edge in the 2D image is approximated by a prolate ellipsoid of radii $4.2 \mu \mathrm{m}, 1.2 \mu \mathrm{m}$ and 1.2 $\mu \mathrm{m}$ (having the equivalent volume of a sphere of radius $a_{\min }=1.82 \mu \mathrm{m}$ ).

The histograms of the distributions of $b$ and of $\nu$ showed in Fig. 4 indicate that the actual semi-minor axis does indeed follow a gamma PDF, but the axial ratio can vary in a large range of values (from 1 up to 7 ). The histograms of the distributions of the aggregate orientation demonstrate that the rouleaux spend more of their time quasi-aligned with the flow streamlines, as observed in the pioneer work of Goldsmith. ${ }^{14}$ Indeed, Fig. 4(d) indicates that more than $39 \%$ of the aggregates in both blood samples have orientations $\alpha=0^{\circ} \pm 10^{\circ}$, whereas, if the distribution were random, $16 \%$ of the aggregates would have this orientation. It is interesting to notice that fitting the $i B S C$ as a function of $\beta$ was sufficient to estimate 
satisfactorily the main orientation of aggregates, even if the aggregates are not perfectly aligned.

Another interesting aspect concerning the actual orientation of aggregates (rf. Fig. 4(d)) is the fact that the distribution of orientations is similar for several studied blood samples (data not shown for several blood samples). A deeper knowledge of the aggregate orientation under various flow conditions (simple shear or tubular flow) would be useful to further develop the anisotropic EMTLMA for estimating QUS parameters. For instance, one could consider using a priori information on the distribution of aggregate orientation for the anisotropic EMTLMA modeling, since the discrepancy between theoretical and simulated BSCs when considering complex aggregating configurations is mainly due to the assumption of perfectly aligned aggregates (as discussed in the Section VA).

\section{Open questions to interpret the anisotropic ultrasonic backscattering from}

\section{sheared aggregating blood}

Several ultrasonic experiments ${ }^{16,17,31,32}$ have been conducted to evaluate the angulardependence of backscattering of sheared RBC suspensions. At low shear rates promoting the highest level of aggregation, the BSC magnitude is highest when the acoustic beam is quasi-perpendicular to the velocity direction (see Figs. 3 and 5 in Ref. ${ }^{16}$ for shear rate $2 \mathrm{~s}^{-1}$, Fig. 7 in Ref. ${ }^{17}$ for shear rate $2 \mathrm{~s}^{-1}$, and Fig. 3 in Ref. ${ }^{31}$ for shear rate $3 \mathrm{~s}^{-1}$ ). Our numerical simulations from complex aggregating configurations (Fig. 5) are in good agreement with these experiments. The anisotropic ultrasound signature ( $i B S C$ as a function of $\beta$ ) could also 
be used to obtain the main orientation of the aggregate structures with the flow streamlines (as discussed previously in subsection VC).

However, at moderate or high shear rates, the BSC magnitude (or the Doppler backscattered power) was also found to be the smallest when the acoustic beam is quasi-perpendicular to the velocity direction (see Fig. 6 in Ref. ${ }^{32}$ in tubular flow for shear rates $17-51 \mathrm{~s}^{-1}$, see Fig. 3 in Ref. ${ }^{31}$ in Couette flow for shear rates $25-140 \mathrm{~s}^{-1}$ ). This anisotropic ultrasound signature in U-shape is difficult to interpret, because it can be attributed to specific size and shape of RBC aggregates as simulated from the smallest standard deviation $\sigma_{b} / a=0.18$ in Fig. 3c, or as hypothesized by Allard et al. ${ }^{32}$ with cone-shaped structures. It may also be due to the shear-induced anisotropic microstructure, as observed in sheared suspensions of hard-spheres by Lombard et al. ${ }^{33}$ Indeed, the spatial organisation (i.e., the microstructure) of hard spheres in sheared concentrated suspensions shows a lower probability of finding pair of particles close to the velocity direction. ${ }^{34}$ This region depleted in particles shows a higher ordering and thus influences greatly the magnitude of BSC and structure factor in the corresponding direction in the frequency domain. ${ }^{33}$ If one considers the aggregate structures as effective scatterers, this region depleted in effective scatterers may explain the anisotropic ultrasound signature in U-shape reported in Refs. ${ }^{31}$ and $^{32}$ at moderate or high shear rates. The numerical simulations performed in this study do not consider the shearinduced anisotropic microstructure, which is not yet understood in RBC suspensions. ${ }^{35}$ In future experimental studies, exploring the use of a confocal microscope with ghost RBCs (i.e., optically visible RBCs with no hemoglobin) to determine the microstructure, size and shape of RBC aggregates within larger microfluidic shearing systems could provide invalu- 
able information to fully understand the ultrasonic backscattering from anisotropic RBC aggregates.

\section{E. Concluding remarks}

The present study contributes to the understanding of ultrasonic scattering from aggregated RBCs and to the practical assessment of RBC aggregate size towards the monitoring of inflammatory response in circulatory diseases. The following contributions were made in this paper.

1. An anisotropic formulation of the EMTLMA was proposed to predict the angulardependent BSC from aligned prolate-shaped aggregates, assuming that the prolateshaped aggregates are perfectly aligned with the flow streamlines. Specifically, the anisotropic EMTLMA considers the correlation among effective prolate ellipsoids (i.e., among aggregates) by using the anisotropic structure factor.

2. The anisotropic EMTLMA model, combined with numerical simulations taking into account the joint PDF $p(b, \nu, \alpha)$, makes it possible to study the influence of specific aggregate structure (polydispersity in terms of size, anisotropic aggregate shape and/or orientation) on the BSC.

3. This numerical study sheds new light on the angular-dependent BSC data to be used for a practical evaluation of RBC aggregate structures. The isotropic EMTLMA that uses only the BSC perpendicular to the flow streamlines (which is the measurement available in a conventional practical approach) presents important deviations from 
the real aggregate size distribution. Whereas the isotropic EMTLMA that uses the averaged BSCs over different insonification directions provide satisfactory estimates. This shows that, for a practical evaluation of RBC aggregate structures, the use of averaged BSCs over different insonification directions can significantly improve the estimation of aggregate structural parameters, even if the model is biased. Overall, the anisotropic EMTLMA estimates the scatterer volume distribution more accurately than the isotropic EMTLMA; but further simulations with complex aggregating configurations (with more varied sizes and orientations) should be conducted to further evaluate the added value of anisotropic modeling for practical assessment of RBC structural features.

\section{ACKNOWLEDGMENTS}

This work was supported by the financial support of Grant No. ANR-15-CE19-0017 and No. ANR-10-LABX-0092, and the Colombian department of Science and technology Colciencias.

\section{REFERENCES}

${ }^{1}$ F. L. Lizzi, M. Ostromogilsky, E. J. Feleppa, M. C. Rorke and M. M. Yaremko, "Relationship of ultrasonic spectral parameters to features of tissue microstructure," IEEE transactions on ultrasonics, ferroelectrics, and frequency control 34(3), 319-329 (1987). 
${ }^{2}$ H. Kitamura, B. Sigel, J. Machi, E. J. Feleppa, J. Sokil-Melgar and J. Justin, "Roles of hematocrit and fibrinogen in red cell aggregation determined by ultrasonic scattering properties," Ultrasound in medicine \& biology 21(6), 827-832 (1995).

${ }^{3}$ Y. Kurokawa, H. Taki, S. Yashiro, K. Nagasawa, Y. Ishigaki, and H. Kanai, "Estimation of size of red blood cell aggregates using backscattering property of high-frequency ultrasound: In vivo evaluation," Japanese Journal of Applied Physics 55(07KF12), 1-8 (2016).

${ }^{4}$ L. Y. L. Mo and R. S. C. Cobbold, "Theoretical models of ultrasonic scattering in blood," in Ultrasonic Scattering in Biological Tissues, edited by K. K. Shung and G. A. Thieme (CRC, Boca Raton, FL, 1993), Chap. 5, pp. 125-170.

${ }^{5}$ F. T. Yu and G. Cloutier, "Experimental ultrasound characterization of red blood cell aggregation using the structure factor size estimator," The Journal of the Acoustical Society of America 122(1), 645-656 (2007).

${ }^{6}$ F. T. Yu, E. Franceschini, B. Chayer, J. K. Armstrong, H. J. Meiselman, and G. Cloutier, "Ultrasonic parametric imaging of erythrocyte aggregation using the structure factor size estimator," Biorheology 46(4), 343-363 (2009).

${ }^{7}$ R. K. Saha, E. Franceschini, and G. Cloutier, "Assessment of accuracy of the structurefactor-size-estimator method in determining red blood cell aggregate size from ultrasound spectral backscatter coefficient," The Journal of the Acoustical Society of America 129(4), 2269-2277 (2011). 
${ }^{8}$ P. Gyawali, D. Ziegler, J.-F. Cailhier, A. Denault, and G. Cloutier, "Quantitative measurement of erythrocyte aggregation as a systemic inflammatory marker by ultrasound Imaging: a systematic review author links open overlay panel," Ultrasound in medicine \& biology 44(7), 1303-1317 (2018).

${ }^{9}$ E. Franceschini, B. Metzger, and G. Cloutier, "Forward problem study of an effective medium model for ultrasound blood characterization," IEEE transactions on ultrasonics, ferroelectrics, and frequency control 58(12), 2668-2679 (2011).

${ }^{10}$ R. De Monchy, J. Rouyer, F. Destrempes, B. Chayer, G. Cloutier, and E. Franceschini, "Estimation of polydispersity in aggregating red blood cells by quantitative ultrasound backscatter analysis," The Journal of the Acoustical Society of America 143(4), 22072216 (2018).

${ }^{11}$ R. de Monchy, B. Chayer, G. Cloutier, and E. Franceschini, "Effective medium theory combined with a polydisperse structure factor model for characterizing red blood cell aggregation," in 2016 IEEE International Ultrasonics Symposium (IUS), IEEE (2016), pp. 1-4.

${ }^{12} \mathrm{R}$. de Monchy, "Development and assessment of effective medium theory combined with polydisperse structure factor for the ultrasonic characterization of erythrocyte aggregation," Ph.D. thesis, Aix-Marseille, 2016.

${ }^{13}$ E. Franceschini, R. K. Saha, and G. Cloutier, "Comparison of three scattering models for ultrasound blood characterization," IEEE transactions on ultrasonics, ferroelectrics, and frequency control 60(11), 2321-2334 (2013). 
${ }^{14}$ H. L. Goldsmith, "The microrheology of red blood cell suspensions," The Journal of general physiology 52(1), 5-28 (1968).

${ }^{15}$ O. Baskurt, B. Neu, and H. J. Meiselman, Red blood cell aggregation, Chap. 3, 31-62 (CRC Press, Boca Raton, FL).

${ }^{16}$ C. Guilbert, F. Yu, and G. Cloutier, "New observations on the anisotropy of ultrasound blood backscatter as a aunction of frequency and shear rate," in 2007 IEEE International Ultrasonics Symposium (IUS), IEEE (2007), pp. 1013-1016.

${ }^{17}$ J. Garcia-Duitama, B. Chayer, A. Han, D. Garcia, M. L. Oelze, and G. Cloutier, "Experimental application of ultrafast imaging to spectral tissue characterization," Ultrasound in medicine \& biology 41(9), 2506-2519 (2015).

${ }^{18}$ R. de Monchy, F. Destrempes, R. Saha, G. Cloutier, and E. Franceschini, "Coherent and incoherent ultrasound backscatter from cell aggregates," The Journal of the Acoustical Society of America 140(3), 2173-2184 (2016).

${ }^{19}$ M. F. Insana and D. G. Brown, "Acoustic scattering theory applied to soft biological tissues", in Ultrasonic Scattering in Biological Tissues, edited by K. K. Shung and G. A. Thieme (CRC, Boca Raton, FL, 1993), Chap. 4, pp. 76-124.

${ }^{20}$ N. Saitô and Y. Ikeda, "The light scattering by non-spherical particles in solutions," Journal of the Physical Society of Japan 6(5), 305-308 (1951).

${ }^{21} \mathrm{M}$. Wertheim, "Exact solution of the percus-yevick integral equation for hard spheres," Physical Review Letters 10(8), 321 (1963). 
${ }^{22}$ E. Franceschini and R. Guillermin, "Experimental assessment of four ultrasound scattering models for characterizing concentrated tissue-mimicking phantoms," The Journal of the Acoustical Society of America 132(6), 3735-3747 (2012).

${ }^{23}$ R. Mehri, C. Mavriplis, and M. Fenech, "Design of a microfluidic system for red blood cell aggregation investigation," Journal of biomechanical engineering 136(6), 064501 (2014).

${ }^{24}$ R. Mehri, E. Niazi, C. Mavriplis, and M. Fenech, "An automated method for dynamic red blood cell aggregate detection in microfluidic flow," Physiological measurement 39(1), 01NT02 (2018).

${ }^{25}$ I. Fontaine, D. Savery, and G. Cloutier, "Simulation of ultrasound backscattering by red cell aggregates: Effect of shear rate and anisotropy," Biophysical Journal 82(4), 1696-1710 (2002).

${ }^{26}$ D. Savery and G. Cloutier, "Effect of red cell clustering and anisotropy on ultrasound blood backscatter: a monte carlo study," IEEE Transactions on Ultrasonics, Ferroelectrics, and Frequency Control 52(1), 94-103 (2005).

${ }^{27}$ R. K. Saha and G. Cloutier, "Monte carlo study on ultrasound backscattering by threedimensional distributions of red blood cells," Physical review E 78(6), 061919 (2008).

${ }^{28}$ Q. W. Guerrero, I. M. Rosado-Mendez, L. C. Drehfal, H. Feltovich, and T. J. Hall, "Quantifying backscatter anisotropy using the reference phantom method," IEEE transactions on ultrasonics, ferroelectrics, and frequency control 64(7), 1063-1077 (2017).

${ }^{29}$ See supplementary material at [URL that will be inserted by AIP] for Tables showing the mean and standard deviations of the QUS parameters for all aggregating configurations. 
${ }^{30}$ V. Claveria, C. Wagner, and P. Connes, "Aggregating and blood flow in health and disease", in Dynamics of blood cell suspensions in microflows, edited by A. Villat and M. Abkarian (CRC, Boca Raton, FL, 2020), Chap. 6, pp. 183-213.

${ }^{31}$ J. Rouyer, L. Chinchilla, O. Lombard, and E. Franceschini, "Characterizing the erythrocyte aggregation using the anisotropy of ultrasonic backscatter," in 2018 IEEE International Ultrasonics Symposium (IUS), IEEE (2018), pp. 1-4.

${ }^{32}$ L. Allard, G. Cloutier, and L.-G. Durand, "Effect of the insonification angle on the doppler backscattered power under red blood cell aggregation conditions," IEEE transactions on ultrasonics, ferroelectrics, and frequency control 43(2), 211-219 (1996).

${ }^{33}$ O. Lombard, J. Rouyer, E. Debieu, F. Blanc, and E. Franceschini, "Ultrasonic backscattering and microstructure in sheared concentrated suspensions," The Journal of the Acoustical Society of America 147(3), 1359-1367 (2020).

${ }^{34}$ F. Blanc, E. Lemaire, A. Meunier, and F. Peters, "Microstructure in sheared non-brownian concentrated suspensions," Journal of rheology 57(1), 273-292 (2013).

${ }^{35}$ L. Mountrakis, E. Lorenz, and A. G. Hoekstra, "Scaling of shear-induced diffusion and clustering in a blood-like suspension," Europhysics Letters 11414002 (2016). 


\section{FIGURE CAPTIONS}

Figure 1. (Color online) Schematic drawing of perfectly aligned prolate ellipsoids.

Figure 2. (Color online) (a) Typical examples of simulated $B S C_{\text {sim }}$ for perfectly aligned with $\phi=30 \%, \bar{b}=2.5 a=6.88 \mu \mathrm{m}, \sigma_{b} / a=0.75$ and $\nu_{f}=2$. The vertical dashed line indicates the frequency of $42 \mathrm{MHz}$. (b) Corresponding $i B S C$ (symbols) in the 10-42 MHz frequency bandwidth as a function of different insonification angles $\beta$. Also plotted in dashed lines are the quadratic function $h(\beta)$ used to estimate the main aggregate orientation.

Figure 3. (Color online) (a) and (b) Comparison between simulated $B S C_{\text {sim }}$ (symbols) and theoretical $B S C_{\text {aniso }}$ (solid lines) at insonification angles $\beta=0^{\circ}$ and $30^{\circ}$ for aligned prolate ellipsoids with identical mean semi-minor axis $\bar{b} / a=2.5$ of varying $\nu_{f}$ (see panel a) and of varying $\sigma / a$ (see panel b). The hematocrit is $30 \%$. The vertical dashed lines indicate the frequency of $42 \mathrm{MHz}$. (c) Corresponding simulated $i B S C$ (symbols) in the 10-42 MHz frequency bandwidth. Also plotted in dashed lines are the quadratic function $h(\beta)$ used to estimate the main aggregate orientation.

Figure 4. (a) Typical example of RBC aggregates flowing in two-fluid flow microfluidic shearing system. The aggregate contours are in green dashed lines and the corresponding fitted ellipses are in red solid lines. (b), (c), (d) and (e) Histogram distributions 
of the semi-minor axis, axial ratio, orientation angle and equivalent radius for the two blood samples studied.

Figure 5. (Color online) (a) Comparison between simulated $B S C_{\text {sim }}$ (symbols) and theoretical $B S C_{\text {aniso }}$ (solid lines) at hematocrit $\phi=10 \%$ for complex aggregating configuration with blood sample A. (b) Same as (a) at hematocrit $\phi=30 \%$. (c) Corresponding simulated $i B S C$ (symbols) in the $10-42 \mathrm{MHz}$ frequency bandwidth. Also plotted in dashed lines are the fitted quadratic function $h(\beta)$, its maximum being indicated by the symbol $\mathrm{x}$.

Figure 6. (Color online) Comparisons between actual and estimated histograms of $r_{\mathrm{eq}}^{3} p\left(r_{\mathrm{eq}}\right)$ using the anisotropic or isotropic EMTLMA for the perfectly aligned prolate ellipsoids $\bar{b} / a=2.5, \nu_{f}=2$ and varying $\sigma_{b}$.

Figure 7. (Color online) Comparisons between actual and estimated histograms of $r_{\text {eq }}^{3} p\left(r_{\text {eq }}\right)$ using the anisotropic or isotropic EMTLMA for hematocrit of $10 \%$ when considering the complex aggregating configurations with both blood samples A and B.

Figure 8. (Color online) Simulated $B S C_{\text {sim }}$ for the complex aggregation configuration using the aggregate features of blood sample $\mathrm{A}$ with $\beta=0^{\circ}$ (losange symbols) and $\beta=30^{\circ}$ (square symbols). The hematocrit is equal to 10\%. Different joint PDFs are considered: $p(b, \nu, \alpha), p\left(b, \nu_{f}, \alpha\right), p\left(b, \nu, \alpha_{f}\right)$ and $p\left(b, \nu_{f}, \alpha_{f}\right)$. 
Chinchilla et al.

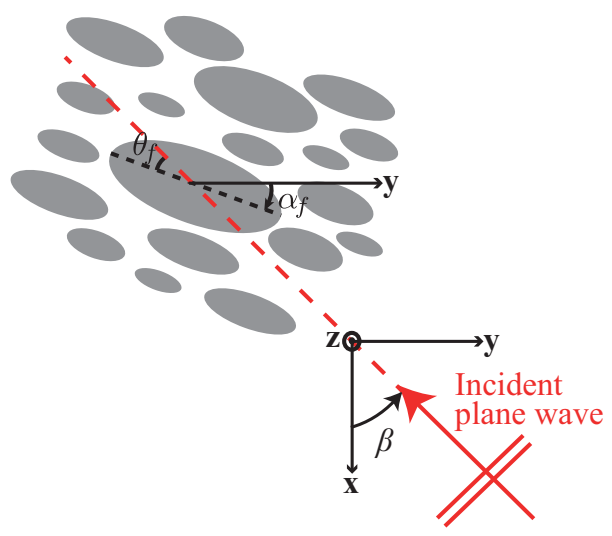

FIG. 1. (Color online) Schematic drawing of perfectly aligned prolate ellipsoids. 

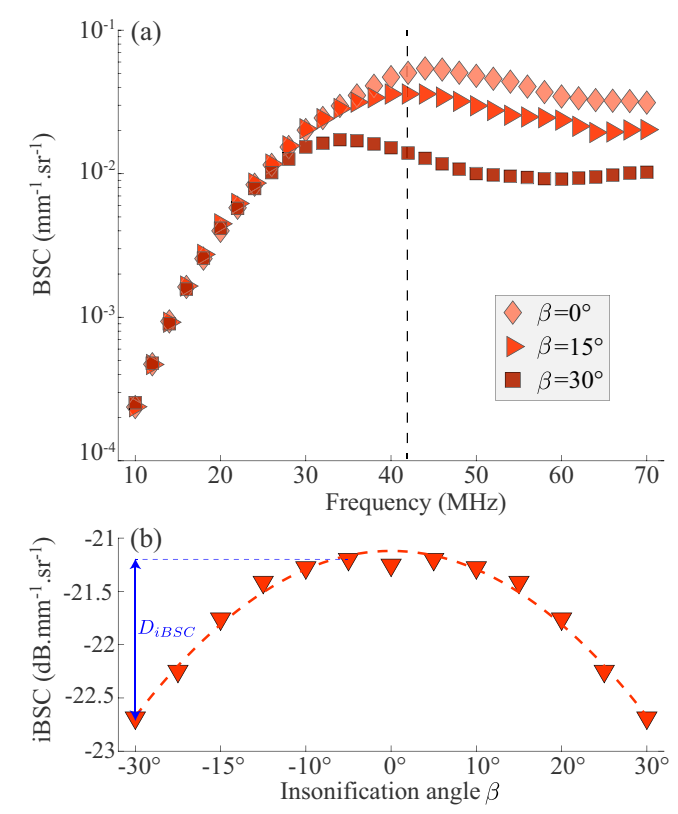

FIG. 2. (Color online) (a) Typical examples of simulated $B S C_{\text {sim }}$ for perfectly aligned with $\phi=30 \%$, $\bar{b}=2.5 a=6.88 \mu \mathrm{m}, \sigma_{b} / a=0.75$ and $\nu_{f}=2$. The vertical dashed line indicates the frequency of 42 MHz. (b) Corresponding $i B S C$ (symbols) in the 10-42 MHz frequency bandwidth as a function of different insonification angles $\beta$. Also plotted in dashed lines are the quadratic function $h(\beta)$ used to estimate the main aggregate orientation. 

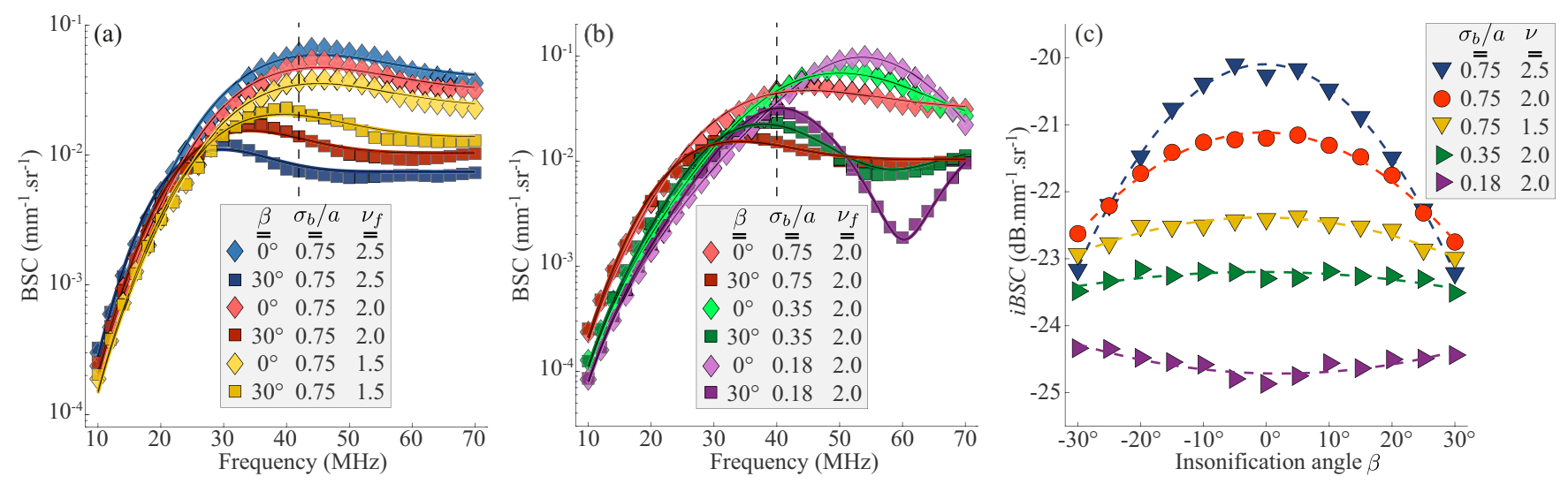

FIG. 3. (Color online) (a) and (b) Comparison between simulated $B S C_{\text {sim }}$ (symbols) and theoretical $B S C_{\text {aniso }}$ (solid lines) at insonification angles $\beta=0^{\circ}$ and $30^{\circ}$ for aligned prolate ellipsoids with identical mean semi-minor axis $\bar{b} / a=2.5$ of varying $\nu_{f}$ (see panel a) and of varying $\sigma / a$ (see panel b). The hematocrit is $30 \%$. The vertical dashed lines indicate the frequency of $42 \mathrm{MHz}$. (c) Corresponding simulated $i B S C$ (symbols) in the 10-42 $\mathrm{MHz}$ frequency bandwidth. Also plotted in dashed lines are the quadratic function $h(\beta)$ used to estimate the main aggregate orientation. 
a)

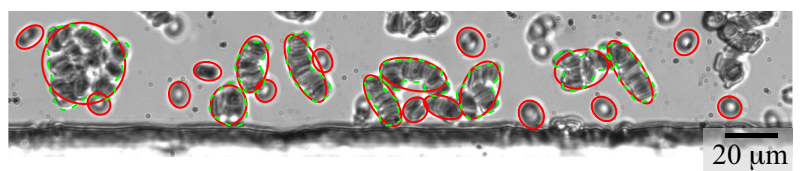

b)
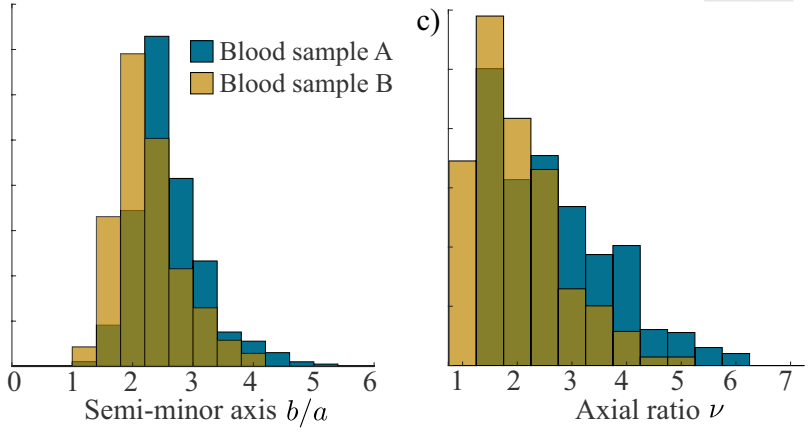

d)
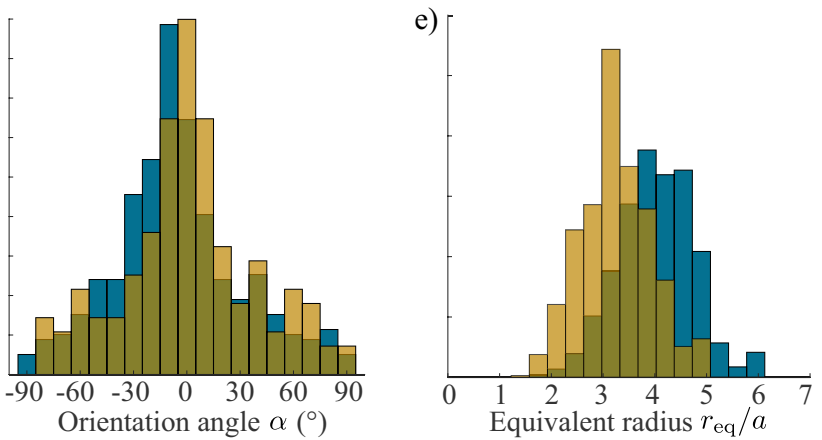

FIG. 4. (Color online) (a) Typical example of RBC aggregates flowing in two-fluid flow microfluidic shearing system. The aggregate contours are in green dashed lines and the corresponding fitted ellipses are in red solid lines. (b), (c), (d) and (e) Histogram distributions of the semi-minor axis, axial ratio, orientation angle and equivalent radius for the two blood samples studied. 

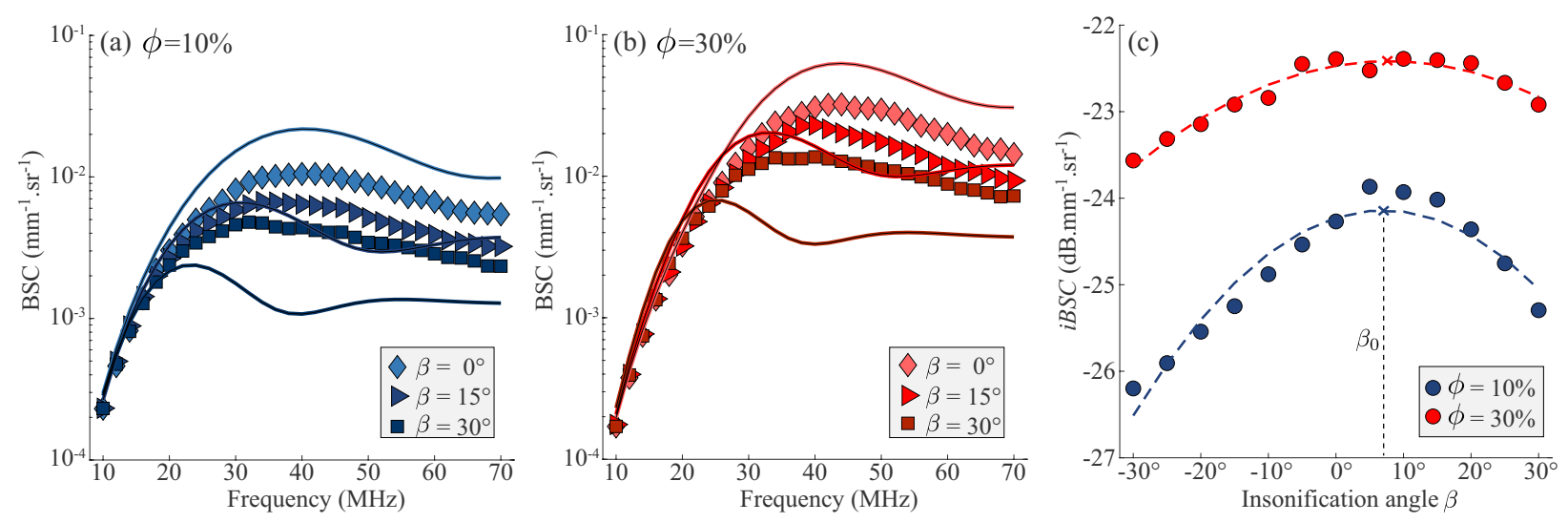

FIG. 5. (Color online) (a) Comparison between simulated $B S C_{\text {sim }}$ (symbols) and theoretical $B S C_{\text {aniso }}$ (solid lines) at hematocrit $\phi=10 \%$ for complex aggregating configuration with blood sample A. (b) Same as (a) at hematocrit $\phi=30 \%$. (c) Corresponding simulated $i B S C$ (symbols) in the 10-42 MHz frequency bandwidth. Also plotted in dashed lines are the fitted quadratic function $h(\beta)$, its maximum being indicated by the symbol $\mathrm{x}$. 

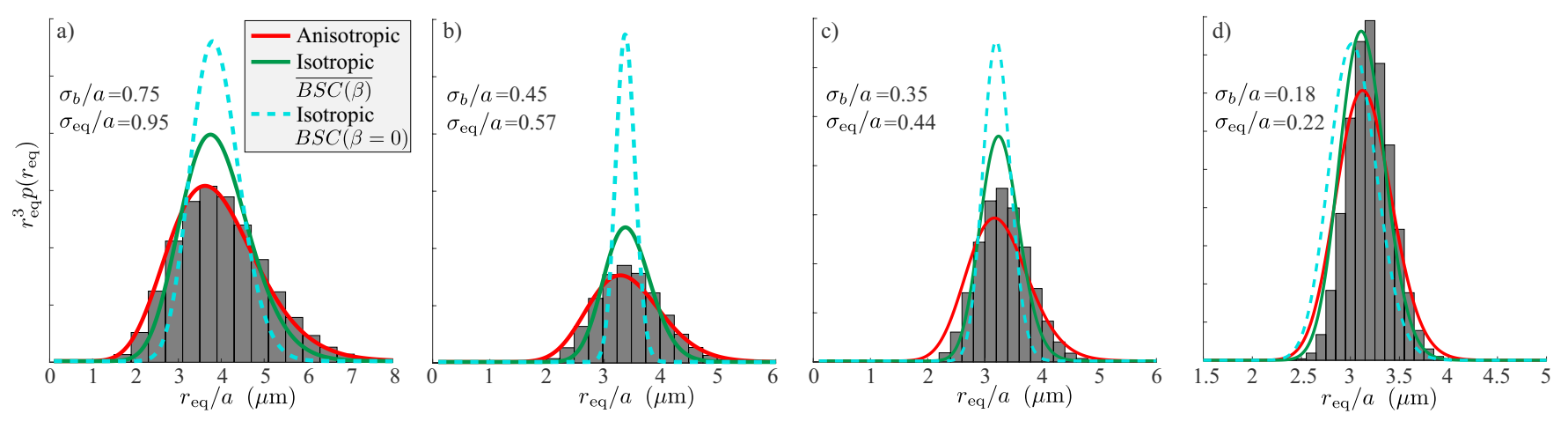

FIG. 6. (Color online) Comparisons between actual and estimated histograms of $r_{\text {eq }}^{3} p\left(r_{\text {eq }}\right)$ using the anisotropic or isotropic EMTLMA for the perfectly aligned prolate ellipsoids $\bar{b} / a=2.5, \nu_{f}=2$ and varying $\sigma_{b}$. 
Chinchilla et al.

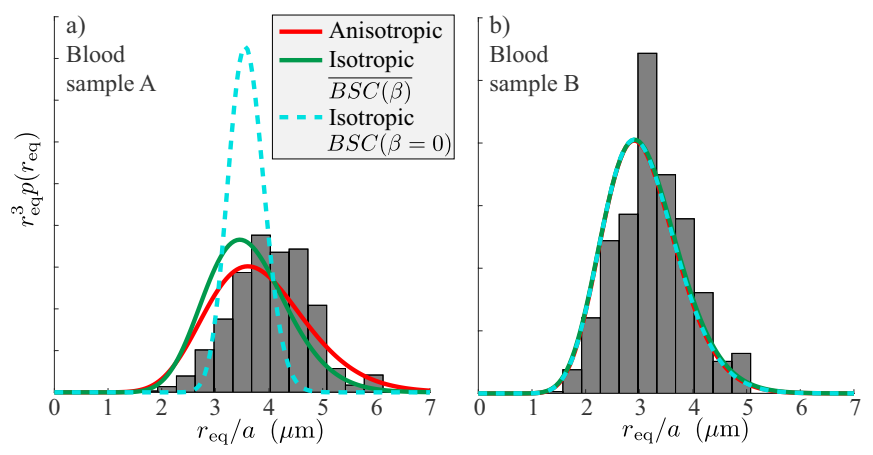

FIG. 7. (Color online) Comparisons between actual and estimated histograms of $r_{\mathrm{eq}}^{3} p\left(r_{\mathrm{eq}}\right)$ using the anisotropic or isotropic EMTLMA for hematocrit of $10 \%$ when considering the complex aggregating configurations with both blood samples A and B. 


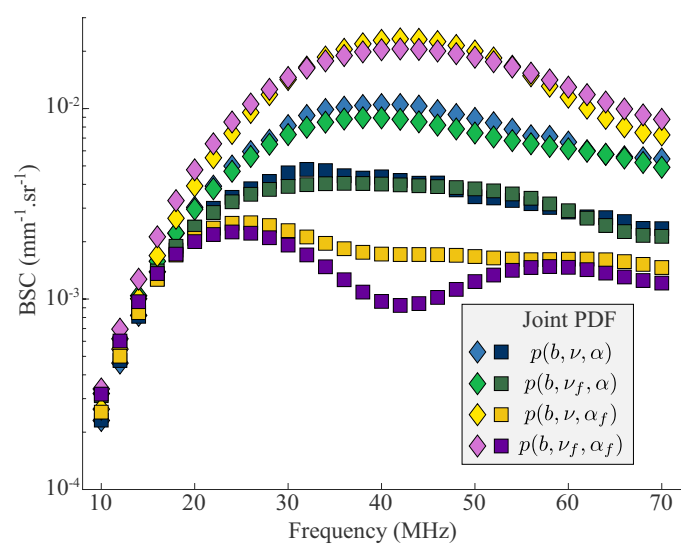

FIG. 8. (Color online) Simulated $B S C_{\text {sim }}$ for the complex aggregation configuration using the aggregate features of blood sample $\mathrm{A}$ with $\beta=0^{\circ}$ (losange symbols) and $\beta=30^{\circ}$ (square symbols). The hematocrit is equal to $10 \%$. Different joint PDFs are considered: $p(b, \nu, \alpha), p\left(b, \nu_{f}, \alpha\right), p\left(b, \nu, \alpha_{f}\right)$ and $p\left(b, \nu_{f}, \alpha_{f}\right)$. 


\section{TABLE CAPTIONS}

Table I. List of symbols.

Table II. The mean of QUS parameters estimated by the anisotropic or isotropic EMTLMA for the aggregating configurations consisting in perfectly aligned prolate ellipsoids at hematocrit $\phi=30 \%$. Also given are the corresponding goodness-of-fit $\mathrm{R}^{2}$.

Table III. The mean values of QUS parameters estimated by the anisotropic or isotropic

EMTLMA for hematocrits of $10 \%$ and $30 \%$ when considering the complex aggregating configurations with blood sample A (see lines 1 and 2) and sample B (see lines 3 and 4). Also given are the corresponding goodness-of-fit $\mathrm{R}^{2}$. 
TABLE I. List of symbols

\begin{tabular}{|c|c|}
\hline$a$ & radius of red blood cell \\
\hline$b$ & semi-minor axis of prolate ellipsoid \\
\hline $\bar{b}$ & mean semi-minor axis of prolate ellipsoid \\
\hline$B S C_{\text {aniso }}$ & backscatter coefficient of isotropic EMTLMA \\
\hline$B S C_{\text {iso }}$ & backscatter coefficient of anisotropic EMTLMA \\
\hline$F$ & ellipsoidal form factor \\
\hline$k$ & wavenumber \\
\hline$m$ & number density of effective prolate ellipsoids \\
\hline$p(b)$ & probablity density function of semi-minor axis \\
\hline$r$ & radius of spherical aggregate \\
\hline$r_{\mathrm{eq}}$ & sphere radius of equivalent \\
\hline & volume to that of prolate ellipsoid \\
\hline$S$ & structure factor \\
\hline$V$ & aggregate volume \\
\hline$\alpha$ & angle between major axis of prolate ellipsoid \\
\hline & and $\mathbf{y}$ axis \\
\hline$\beta$ & angle defining the insonification angle \\
\hline$\gamma_{\kappa}$ & $\begin{array}{l}\text { relative contrast in compressibility } \\
43\end{array}$ \\
\hline$\gamma_{\rho}$ & relative contrast in density \\
\hline
\end{tabular}


TABLE II. The mean of QUS parameters estimated by the anisotropic or isotropic EMTLMA for the aggregating configurations consisting in perfectly aligned prolate ellipsoids at hematocrit $\phi=30 \%$. Also given are the corresponding goodness-of-fit $\mathrm{R}^{2}$.

\begin{tabular}{|c|c|c|c|c|c|c|c|c|c|}
\hline & \multicolumn{3}{|c|}{ Actual aggregates parameters } & \multicolumn{6}{|c|}{ QUS parameters estimated by EMTLMA } \\
\hline & \multirow[b]{2}{*}{$\left(\bar{b} / a, \sigma_{b} / a, \nu_{f}\right)$} & \multirow[b]{2}{*}{$\left(\bar{r}_{\mathrm{eq}} / a, \sigma_{\mathrm{eq}} / a\right)$} & \multirow[b]{2}{*}{$\mathrm{D}_{i B S C}$} & \multicolumn{2}{|l|}{ Anisotropic } & \multicolumn{2}{|c|}{ Isotropic $(\overline{B S C(\beta)})$} & \multicolumn{2}{|c|}{ Isotropic $(B S C(\beta=0))$} \\
\hline & & & & $\left(\bar{b}^{*} / a, \sigma_{b}^{*} / a, \nu_{f}^{*}\right)$ & $\mathrm{R}^{2}$ & $\left(\bar{r}^{*} / a, \sigma_{r}^{*} / a\right)$ & $\mathrm{R}^{2}$ & $\left(\bar{r}^{*} / a, \sigma_{r}^{*} / a\right)$ & $\mathrm{R}^{2}$ \\
\hline 1 & $(2.50,0.75,1.5)$ & $(2.86,0.61)$ & 0.55 & $(2.47,0.75,1.44)$ & 0.97 & $(3.09,0.73)$ & 0.93 & $(3.27,0.61)$ & 0.89 \\
\hline 2 & $(2.50,0.75,2.0)$ & $(3.15,0.95)$ & 1.49 & $(2.45,0.75,1.93)$ & 0.97 & $(3.42,0.75)$ & 0.80 & $(3.64,0.55)$ & 0.60 \\
\hline 3 & $(2.50,0.75,2.5)$ & $(3.39,1.02)$ & 3.06 & $(2.44,0.72,2.57)$ & 0.98 & $(3.55,0.82)$ & 0.67 & $(3.80,0.66)$ & 0.25 \\
\hline 4 & $(2.50,0.45,2.0)$ & $(3.15,0.57)$ & 0.31 & $(2.44,0.49,1.94)$ & 0.98 & $(3.27,0.40)$ & 0.96 & $(3.35,0.17)$ & 0.94 \\
\hline 5 & $(2.50,0.35,2.0)$ & $(3.15,0.44)$ & 0.26 & $(2.46,0.42,1.80)$ & 0.98 & $(3.17,0.33)$ & 0.98 & $(3.16,0.24)$ & 0.97 \\
\hline 6 & $(2.50,0.18,2.0)$ & $(3.15,0.22)$ & 0.46 & $(2.44,0.18,2.03)$ & 0.99 & $(3.08,0.23)$ & 0.99 & $(2.98,0.24)$ & 0.98 \\
\hline
\end{tabular}


TABLE III. The mean values of QUS parameters estimated by the anisotropic or isotropic EMTLMA for hematocrits of $10 \%$ and $30 \%$ when considering the complex aggregating configurations with blood sample A (see lines 1 and 2) and sample B (see lines 3 and 4). Also given are the corresponding goodness-of-fit $\mathrm{R}^{2}$.

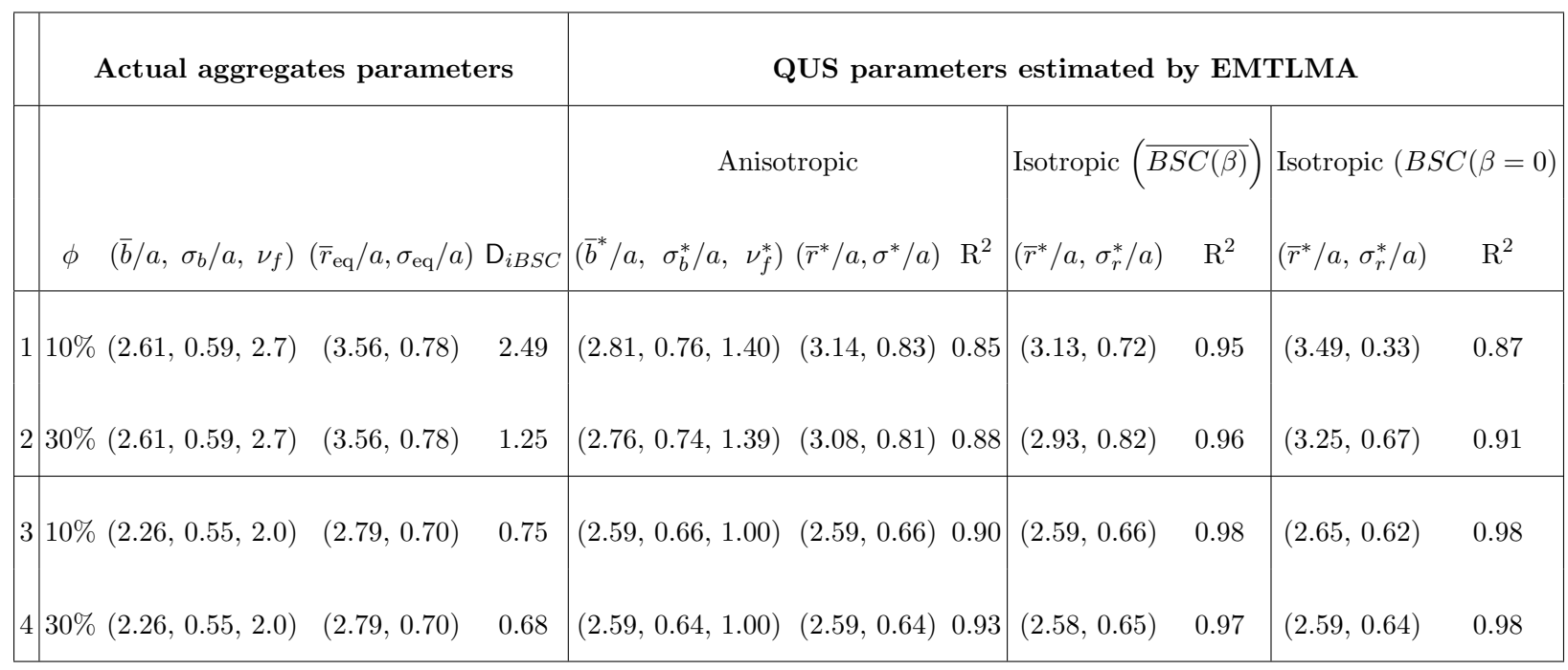

